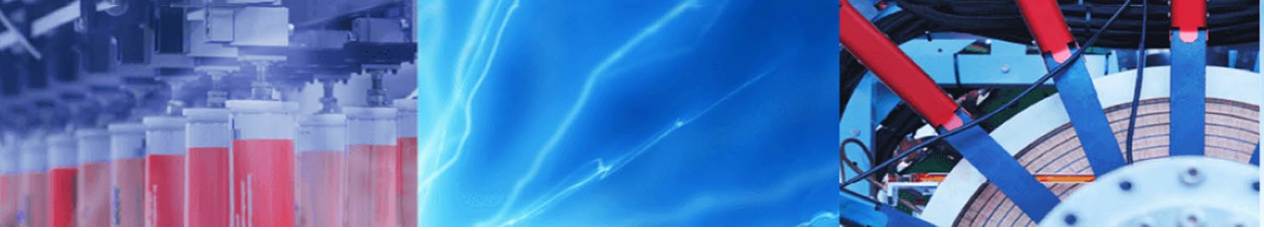

Research Article

\title{
A lattice Boltzmann model for computing compressible two-phase flows with high density ratio
}

\author{
Hossein Yazdi ${ }^{1}$ Mohammad Hassan Rahimian ${ }^{1}$ (I) $\cdot$ Hesameddin Safari ${ }^{2}$
}

Received: 29 August 2019 / Accepted: 9 December 2019 / Published online: 14 December 2019

(c) Springer Nature Switzerland AG 2019

\begin{abstract}
The objective of this article is to present a novel LB method to simulate compressible two-phase liquid-gas flows with two distribution functions which recovers fully compressible Navier-Stokes equations. A lattice Boltzmann framework with two distribution functions is developed in which one of the distribution functions computes the density, momentum and the temperature while the other one recovers the Cahn-Hilliard equation to capture the phase interface. The equilibrium density distribution function is chosen in such a way that the lattice Boltzmann equation recovers the Navier-Stokes equations together with the energy equation using the multiscale Chapman-Enskog expansion. The D1Q5 lattice Boltzmann model is developed to solve various test cases in one dimension including the shock tube problem. The good agreement between the numerical results and exact solutions confirms the accuracy and consistency of the method. It is also shown that the developed method is able to stably compute the compressible two-phase flows with density ratios up to 700 .
\end{abstract}

Keywords Lattice Boltzmann method · Two-phase flow · Compressible flow · Cavitation · Shock-tube

\section{Introduction}

Cavitation is the formation of bubbles in the fluids under certain circumstances. This issue was first addressed in 19th mid-century [1] and was identified as a damaging phenomenon in ship and submarine propellers. Nowadays, the cavitation phenomenon has engaged two groups of engineers and experts in different industries, the first group seeks a method to reduce the occurrence probability of this phenomenon in their manufactured devices to reduce its damages while the second group attempts to generate different forms of the cavitation phenomenon to be utilized in specific applications (e.g. medical applications).

Generally, there are three main issues in the cavitation modeling; firstly, evaporation or distillation phenomena due to the pressure increase or decrease with respect to the saturation pressure, secondly, consideration of the compressibility in the gaseous phase and thirdly, generating a consistent flow field to account for the pressure increase or decrease throughout the computational domain. The issue of the pressure-driven droplet evaporation and distillation is investigated in the previous paper [2]. In the current work, the compressibility of the gaseous phase is studied.

Numerical simulation of the multiphase flows is a challenging task with the key application in the industry and natural phenomena. Some examples of these applications include bubble dynamics, reactive and combustible systems, supersonic dryer apparatus, treatment of cancer cells in medical industries, blood flow through the blood vessels and food industries [3,4].

\footnotetext{
$\triangle$ Mohammad Hassan Rahimian, rahimyan@ut.ac.ir| ${ }^{1}$ Department of Mechanical Engineering, College of Engineering, University of Tehran, Tehran, Iran. ${ }^{2}$ Institute for Computational Modeling in Civil Engineering, Technische Universität Braunschweig, Brunswick, Germany.
} 
In the classical methods for computation of two-phase fluid flows, the proposed approaches for resolving the phase interface can be divided into two categories; the sharp interface method (SIM) and the diffuse interface method (DIM) [4]. In SIMs, two different methods of interface capturing or interface tracking may be used to resolve the phase interface. The volume of fluid (VOF) [5-7], and the level set (LS) [8-10] are among the most well-known interface capturing methods and the method of Tryggvason [11] is one of the mostly used for the interface tracking methods. The major drawbacks of the interface tracking methods are high complexity, high computational costs and instability problems where substantial interfacial deformations occur [12]. Recently, Chang and Liou [13] developed a model for stratified flows based on SIM which has good capability in modeling gas-liquid compressible flows.

In DIM, the interface is considered as a physically (or numerically) diffused region. The interfacial area between the two phases can be justified as a kind of artificial mixtures generated due to the numerical diffusion. This method is also divided into two categories in the context of the compressible two-phase flow simulation: the methods based on Eulers equations [13-18] and the methods based on the multicomponent equations [19]. The methods based on the Eulers equations are limited to simple physical models and simple equation of states. Furthermore, they suffer from the lack of accuracy in terms of the internal energy and temperature at the interface.

The most comprehensive model in this field is the seven-equation model proposed by Saurel and Abgrall [20]. The seven equations include two continuity, two momentum, two energy equations together with one equation for solving the volume fractions of each phases. This model computes two separate pressures and velocities and has more efficiency when the velocity difference between the two phases is of interest. Due to the nonconservative terms appear in the momentum, energy and the volume fraction equations, the application of this model seems to be challenging. Several attempts have been made in using this method [21-25]. Another alternative is the single velocity six-equation model. This model was proposed by Kapila et al. [26] for the first time. They assumed the zero relaxation time for the velocity field. As this model uses a single velocity formulation for the whole domain, it has a lower diffusivity throughout the interfacial regions compared to the seven-equation model.

Another suitable model for simulating compressible multiphase flows is the reduced five-equation model which is also known as the Kapila model $[26,27]$. Different works have been published during the recent years in order to propose an efficient numerical scheme for solving the five-equations model. [3, 28-31]. For instance, Ansari et al. [32] performed the numerical simulation of twophase flows with a five-equation two-fluid model. In order to improve the accuracy, they employed the MUSCL-Hancock scheme for the Godunov method and the secondorder HLLC Riemann solver. One of the most important advantages of this method is the lower numerical oscillations as well as the reduction in numerical diffusion.

The lattice Boltzmann method (LBM) is a mesoscopic method which lies in between the microscopic and macroscopic approaches. In this method, the evolution of a particle (or density) distribution function is obtained by solving a Boltzmann equation over a computational grids called lattice. The macroscopic quantities of the flow (e.g. density and velocity) are computed by taking the zeroand first-order moments of the calculated distribution function. Due to its mesoscopic nature, the LBM has been successfully applied to numerous two-phase flow simulations over the last two decades [33-38]. Despite the recent advances in the lattice Boltzmann (LB) simulation of multiphase flows, all these works are limited to incompressible flows. To the best of our knowledge, the LB computation of the compressible two-phase flow has not yet been accomplished.

In the common LBM framework, the equilibrium distribution function $\left(f_{i}^{e q}\right)$ polynomial is typically obtained by applying the Taylor expansion of the Boltzmann-Maxwellian distribution function up to the second-order in velocity. Thus, this expansion is valid for small Mach numbers. Otherwise, the truncation error resulted from the Taylor series expansion would be very large. This condition limits the application of the common lattice Boltzmann method to the incompressible flows. Various models have been proposed to extend the LBM for the computation of compressible flows.

Studies have shown that the two-phase shock problem has not been simulated in LBM and cannot be used in literature. Therefore, first, the simulation methods for compressible single phase in LBM are briefly presented and then the simulations of two phase problems are explained.

Guangwu et al. [39] proposed a 2D 9-bit model with two energy levels. Their model can recover Euler equations. In this model, the lattice was considered similar to that of the standard D2Q9. The difference is that there are two particles of $f_{i A}$ and $f_{i B}(\mathrm{i}=1 \ldots 8)$ in any direction of the lattice except for the rest position ( $i=0)$. Thus, there are totally 17 particles. These 17 particles are categorized in three energy levels.

Shi [40] developed a 2D 9-bit model which is able to recover Euler equations. They used the standard D2Q9 lattice and introduced three energy levels: $\epsilon_{D}(i=0)$, $\epsilon_{B}(i=5,6,7,8)$ and $\epsilon_{A}(i=1,2,3,4)$ which leads to the adjustable specific heat ratios. Instead of using collisionstreaming algorithm, the finite difference method of TVD 
was used in this model in order to solve the discretized velocity Boltzmann equation (DVBE).

Kataoka and Tsutahara [41] proved that for the small Knudsen numbers, the discretized velocity Boltzmann equation can approximate the Euler equations throughout the smooth regions. At the stiff regions, the discretized velocity Boltzmann equations may estimate the weak solution of the Euler equations. They have also developed several LBM models for the compressible flows. Their method for the two-dimensional inviscid flows is similar to the model of Shi [40]. Despite the model consists of nine lattice velocities, the lattice structure differs from the common D2Q9 lattices. Furthermore, Kataoka and Tsutahara [42] proposed a two dimensional 16-velocity model for the compressible flows. The $f_{i}^{e q}$ is estimated by a higher order polynomial. Similar to the model of Shi [40], the forward second-order finite difference approach was used to solve the DVBE. They have successfully simulated the shock tube and Couette flow problems. However, their model was prone to the numerical instability when the Mach number increases.

In order to simulate the compressible flows, Sun [43-47] developed an adaptive lattice Boltzmann model. In this model, the lattice speeds are varied by the mean flow velocity and the internal energy. The adaptive lattice Boltzmann model is constructed using different discretized microscopic velocity vectors located symmetrically around the mean flow velocity in the velocity space. This adaptive lattice Boltzmann is capable for simulating the flows with high Mach numbers. However, the relaxation coefficient of the collision operator $(\tau)$ can only be equal to the unity as the discretized microscopic velocities change with the mean velocity vector. Thus, viscosity can not be adjusted by changing $\tau$ and the model is only suitable for the simulation of inviscid flows. Several flows with weak and strong shock waves have been successfully simulated by means of the adaptive lattice Boltzmann model. This approach is a promising method. The equilibrium distribution function is simply defined and not related to the Boltzmann-Maxwellian distribution function, However, it can recover the Navier-Stokes equations. Using this approach, the equilibrium distribution function could be substituted by other functions while the feature of recovering Euler/Navier-Stokes equations is maintained. This idea is significant in developing new models for simulating the compressible flows.

Recently, two other models have been proposed for the computation of compressible flows by means of the LBM which are able to solve compressible flows at high Mach numbers. The first method is the finite difference lattice Boltzmann method proposed by Watari et al. [48-51]. They simulated the supersonic viscous flow in the microchannel by applying compact finite difference method for solving the Boltzmann equation. The second model has been proposed by Qu et al. $[52,53]$ in which the forward finite volume LBM is utilized to solve the compressible problems at high Mach numbers. They simulated the compressible inviscid flow using the finite volume LBM with central differencing scheme over the unstructured lattices.

Recently, He et al. [54] have studied the compressible flow with shock waves in one- to three- dimensional test cases using the dual distribution function. In their model, the density distribution function is used to compute the flow field and the total energy distribution function is used to calculate the temperature field. The discretized forms of the density and the total energy distribution functions are obtained by the Hermite polynomial expansion. In order to improve the accuracy and stability for the shock capturing, the implicit-explicit finite difference scheme is employed.

Since our ultimate goal is to develop a LB model suitable for simulating the two-phase compressible flows which can be applicable for cavitating flows, we briefly review the multiphase LB models with the focus on the phase-change modeling. In the context of LB models for multiphase flows, Gunstensen [55] introduced the first model for the immiscible binary fluid in two dimensions. Later, Shan and Chen $[56,57]$ introduced their well-known multiphase model in which a force term is added to the Boltzmann equation as an intermolecular force. He et al. [58] proposed a model with two distribution functions for modeling the two-phase fluid flows. One of the distribution functions calculates the momentum and pressure while the other is used to compute the index function which is employed to distinguish each phases.

Lee and Lin [59] developed a pressure evolution equation LB model in which the pressure evolution equation is obtained by taking the time derivative from the equation of state for non-ideal gases. This model allows the fluid at the phase interface to be compressible when phase change occurs due to pressurization and depressurization. They simulated a one-dimensional isothermal phasechange process and their results were in good agreement with analytical solutions.

Zhang and Chen [60] proposed a pseudopotential model, for computing thermal multiphase flows which has been claimed to be thermodynamically consistent at the macroscopic level. In this model, the temperature field is calculated by solving the scalar energy transport equation with a finite difference scheme. They simulated the liquid-vapor boiling process, however, only qualitative results were shown and they did not validate the simulation results quantitatively.

Recently, Lee [61, 62] developed a lattice Boltzmann binary-fluid model based on the convective Cahn-Hilliard equation. At the cost of the sophisticated and computationally expensive schemes for the evaluation of 
derivatives, this model eliminates the parasitic currents and maintains the stability for density ratios up to 1000 . Safari et al. [63] extended the Lee's model to simulate the thermal phase-change phenomena consistently over the wide range of density ratios. In this approach, appropriate forcing terms are added to the LB equations of the momentum and phase-field to consistently account for the phase-change process across the phase interface. They validated the proposed model with one-dimensional Stefan problem. Later, Safari et al. [64] extended this approach to take into account the vapor concentration field and relate the evaporation rate to the gradient of vapor concentration at the liquid-vapor interface. They simulated a two-dimensional evaporating droplet in quiescent and convective environments and confirmed the consistency of this approach with the aid of experimental results. Yazdi et al. [2] used the same approach proposed by Safari et al. [63] to present a model to simulate the phase-change phenomena due to the pressurization and depressurization throughout the computational domain.

Mohammadi-Shad et al. [65] modeled the phasechange processes with the similar approach used by Safari et al. [63] and solved the energy equation by employing the sharp-interface energy solver. They validated their method by solving the Stefan problem in one and two dimensions. Furthermore, the problem of a bubble growth and rising inside the superheated liquid has been investigated.

Using the model presented by Safari et al. [63], Begmohammadi et al. [66] simulated the periodic growth and departure of a bubble from a superheated horizontal plate. Sadeghi et al. [67] studied the pool-boiling problem at the high density ratio by extending the method of Safari [63] to three dimensions. They predicted the temperature field in the vicinity of the bubble interface.

As previously mentioned, the numerical simulation of compressible two-phase flows has not yet been conducted using LBM. According to studies by the authors, the simulation of the two-phase shock problem with the LBM method has not been performed so far. In this article, the two-phase compressible method based on LBM is developed and with this method, the two-phase shock problem is solved. In this approach, the intermolecular forcing term (computed using the method of $\mathrm{He}$ et al. [58]) is introduced into the compressible model of Kataoka [42], so that the phase separation can be realized by the model. Moreover, the Cahn-Hilliard equation is solved by a separate distribution function in order to capture the phase interface. In the following sections, the mathematical modeling and discretization of the governing equations are presented. Subsequently, the model is validated using the shock-tube test case. Then a test case with density ratio about 700 is presented.

\section{Mathematical modeling}

\subsection{Governing equations}

The Boltzmann equation for a non-ideal fluid in the presence of intermolecular forces can be written as $[35,58]$

$\frac{D f}{D t} \equiv \frac{\partial f}{\partial t}+\mathbf{c} \cdot \nabla f=-\frac{f-f^{e q}}{\lambda}+\frac{(\mathbf{c}-\mathbf{u}) \cdot \mathbf{F}+\mathbf{G}}{\rho R T} f^{e q}$

where $f$ is the distribution function, $f^{e q}$ is the equilibrium distribution function, $\mathbf{c}$ denotes the microscopic velocity, $G$ represents the gravity force, $\rho$ is the density, $T$ is the temperature, $R$ is the gas constant and $F$ shows the effective intermolecular force.

He et al. [58] pointed that by employing the mean-field approximation for the intermolecular attraction [68], the effective intermolecular force can be computed as follows [58],

$F=-\nabla \psi+\kappa \rho \nabla \nabla^{2} \rho$

The parameter $\kappa$ is related to the inter-molecular pair-wise potential and $\psi$ calculated as follows:

$\psi=P-\rho R T$

in which the pressure $(P)$ is calculated from the van der Waals equation of state:

$P=\frac{\rho R T}{1-b_{\text {van }} \rho}-a_{\text {van }} \rho^{2}$

$a_{\text {van }}$ and $b_{\text {van }}$ are van der Waals Coefficients.

Here, the fully compressible Navier-Stokes equations together with the energy conservation equation are given for more clarification.

$$
\begin{aligned}
& \frac{\partial \rho}{\partial t}+\frac{\partial \rho u_{\alpha}}{\partial x_{\alpha}}=0 \\
& \frac{\partial \rho u_{\alpha}}{\partial t}+\frac{\partial \rho u_{\alpha} u_{\beta}}{\partial x_{\beta}}+\frac{\partial p}{\partial x_{\alpha}}=-\frac{\partial P^{\prime}{ }_{\alpha \beta}}{\partial x_{\beta}} \\
& \frac{\partial \rho\left(b R T+u_{\alpha}^{2}\right)}{\partial t}+\frac{\partial \rho u_{\alpha}\left(b R T+u_{\beta}^{2}\right)+2 p u_{\alpha}}{\partial x_{\alpha}} \\
& =2 \frac{\partial}{\partial x_{\beta}}\left(\lambda \frac{\partial T}{\partial x_{\beta}}-P^{\prime}{ }_{\alpha \beta} u_{\alpha}\right)
\end{aligned}
$$

where

$P_{\alpha \beta}^{\prime}=-\mu\left(\frac{\partial u_{\alpha}}{\partial x_{\beta}}+\frac{\partial u_{\beta}}{\partial x_{\alpha}}-\frac{2}{3} \frac{\partial u_{\mathcal{X}}}{\partial x_{\mathcal{X}}} \delta_{\alpha \beta}\right)-\mu_{\beta} \frac{\partial u_{\mathcal{X}}}{\partial x_{\mathcal{X}}} \delta_{\alpha \beta}$

$(\alpha, \beta, \mathcal{X}=1,2, \ldots, D)$ 
where $t$ is the time, $x_{a}$ is the spatial coordinate, $u_{a}$ is the flow velocity in the $x_{a}$ direction, $\mathrm{D}$ is the number of spatial dimensions, respectively. Note that $b$, in the above expression, is a constant which is related to the specific heat ratio.

$b=\frac{2}{\gamma-1}$

In order to recover the Navier-Stokes equations using the multiscale Chapman-Enskog expansion, the equilibrium distribution function should satisfy the conditions in Eq. (8). It has been shown in the Appendix A of Ref. [48] that employing theses conditions, the Navier-Stokes equations are consistently recovered.

$$
\begin{aligned}
& \sum_{i=1}^{l} f_{i}^{e q}=\rho \\
& \sum_{i=1}^{\prime} f_{i}^{e q} c_{i \alpha}=\rho u_{\alpha} \\
& \sum_{i=1}^{1} f_{i}^{e q} c_{i \alpha} c_{i} \beta=p \delta_{\alpha \beta}+\rho u_{\alpha} u_{\beta} \\
& \sum_{i=1}^{1} f_{i}^{e q}\left(c_{i \alpha}^{2}+\eta_{i}^{2}\right)=\rho\left(b R T+u_{\alpha}^{2}\right) \\
& \sum_{i=1}^{1} f_{i}^{e q}\left(c_{i \beta}^{2}+\eta_{i}^{2}\right) c_{i \alpha}=\rho\left[(b+2) R T+u_{\beta}^{2}\right] u_{\alpha} \\
& \sum_{i=1}^{l} f_{i}^{e q} c_{i \alpha} c_{i \beta} c_{i \mathcal{X}}=\rho\left[R T\left(u_{\alpha} \delta_{\beta \mathcal{X}}+u_{\beta} \delta_{\mathcal{X} \alpha}+u_{\mathcal{X}} \delta_{\alpha \beta}\right)+u_{\alpha} u_{\beta} u_{\mathcal{X}}\right] \\
& \sum_{i=1}^{1} f_{i}^{e q}\left(c_{i \mathcal{X}}^{2}+\eta_{i}^{2}\right) c_{i \alpha} c_{i \beta}=\rho\left\{(b+2) R^{2} T^{2} \delta_{\alpha \beta}+\left[(b+4) u_{\alpha} u_{\beta}+u_{\mathcal{X}}^{2} \delta_{\alpha \beta}\right] R T+u_{\mathcal{X}}^{2} u_{\alpha} u_{\beta}\right\}
\end{aligned}
$$$$
C_{i}= \begin{cases}0 & i=1 \\ v_{1} \cos (\pi i) & i=2,3 \\ v_{2} \cos (\pi i) & i=4,5\end{cases}
$$

where

$$
Z=\frac{1}{1-b_{\text {van }} \rho}-\frac{a_{\text {van }} \rho}{T R}
$$

Note that in above equations, $I=5$ for D1Q5.

It can be shown that the following $f^{e q}$ satisfies the above conditions.

$f_{i}^{e q}=\rho\left(A_{i}+B_{i} u C_{i}\right) \quad i=1,2,3,4,5$

where

$A_{i}=\left\{\begin{array}{lc}\frac{b-Z}{\eta_{0}^{2}} R T & i=1 \\ \frac{1}{2\left(v_{1}^{2}-v_{2}^{2}\right)}\left[-v_{2}^{2}+\left((b-Z) \frac{v_{2}^{2}}{\eta_{0}^{2}}+Z\right) R T+u^{2}\right] & i=2,3 \\ \frac{1}{2\left(v_{2}^{2}-v_{1}^{2}\right)}\left[-v_{1}^{2}+\left((b-Z) \frac{v_{1}^{2}}{\eta_{0}^{2}}+Z\right) R T+u^{2}\right] & i=4,5\end{array}\right.$

$B_{i}= \begin{cases}0 & i=1 \\ \frac{-v_{2}^{2}+(b+2) T+u^{2}}{2 v_{1}^{2}\left(v_{1}^{2}-v_{2}^{2}\right)} & i=2,3 \\ \frac{-v_{1}^{2}(b+2) T+u^{2}}{2 v_{2}^{2}\left(v_{2}^{2}-v_{1}^{2}\right)} & i=4,5\end{cases}$ where $v_{1}, v_{2}$ and $\eta_{0}$ are given nonzero constants.

As depicted in Fig. 1, the D1Q5 lattice Boltzmann model is used for solving the problems in one dimension.

The macroscopic quantities are calculated from the following expressions [42]:

$\rho=\Sigma f_{i}$

$\rho u=\Sigma f_{i} c_{i}$

$\rho\left(b R T+u^{2}\right)=\Sigma f_{i}\left(c_{i}^{2}+\eta_{i}^{2}\right)$

In order to calculate the constant $b$ in the above equations, the specific heat coefficient is required. Since a two-phase fluid flow is considered, the value of the specific heat at

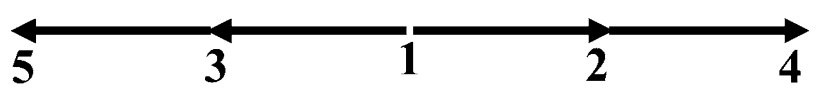

Fig. 1 The D1Q5 model 
each node depends on the phase composition. Thus, the Cahn-Hilliard equation is solved to capture the phase interface and calculate the phase field. The Cahn-Hilliard equation can be solved within a LBM framework. For this purpose, another distribution function (called $h_{\alpha}$ ) is defined as $h_{\alpha}=(\phi / \rho) f_{\alpha}$ and $h_{\alpha}^{e q}=(\phi / \rho) f_{\alpha}^{e} q$, where $\phi$ is the volume fraction of the liquid phase given as follows,

$\phi=\frac{\widetilde{\rho}_{l}}{\rho_{l}}=>\widetilde{\rho}_{G}=(1-\phi) \rho_{G}$

Taking the total derivative from the both side of the equation $h_{\alpha}=(\phi / \rho) f_{\alpha^{\prime}}$ one can easily observe that

$\frac{D}{D t}\left(h_{\alpha} \rho\right)=\frac{D}{D t}\left(\phi f_{\alpha}\right)$

$h_{\alpha} \frac{D \rho}{D t}+\rho \frac{D h_{\alpha}}{D t}=f_{\alpha} \frac{D \phi}{D t}+\phi \frac{D f_{\alpha}}{D t}$

where

$$
\begin{aligned}
\frac{D \rho}{D t} & =\left(\frac{\partial}{\partial t}+\boldsymbol{c}_{\alpha} \cdot \nabla\right) \rho=\left(\boldsymbol{c}_{\alpha}-\boldsymbol{u}\right) \cdot \nabla \rho-\rho \nabla \cdot \mathbf{u} \\
\frac{D f_{\alpha}}{D t} & =\left(\frac{\partial}{\partial t}+\boldsymbol{c}_{\alpha} \cdot \nabla\right) f_{\alpha}=-\frac{1}{\lambda}\left(f_{\alpha}-f_{\alpha}^{e q}\right) \\
& +\frac{1}{c_{s}^{2}}\left(\boldsymbol{c}_{\alpha}-\boldsymbol{u}\right) \boldsymbol{F} \Gamma_{\alpha} \\
\frac{D \phi}{D t} & =\left(\frac{\partial}{\partial t}+\boldsymbol{c}_{\alpha} \cdot \nabla\right) \phi
\end{aligned}
$$

After some algebra, the discretized Boltzmann equation for $h_{\alpha}$ is obtained as follows:

$$
\begin{aligned}
& \frac{D h}{D t}=\frac{h}{\phi} \frac{D \phi}{D t}+\frac{\phi}{\rho} \frac{D f}{D t}-\frac{h}{\rho} \frac{D \rho}{D t}= \\
& \frac{h}{\phi}\left(\left(\frac{\partial}{\partial t}+c_{i} \cdot \nabla\right) \phi\right)+\frac{\phi}{\rho}\left(-\frac{f-f^{e q}}{\lambda}+\frac{f^{e q}}{\rho R T}\left(c_{i}-u\right) F\right) \\
& -\frac{h}{\rho}\left(\left(c_{i}-u\right) \cdot \nabla \rho-\rho \nabla \cdot u\right)= \\
& \frac{h-h^{e q}}{\lambda}+\frac{h}{\phi}\left(\left(\frac{\partial}{\partial t}+c_{i} \cdot \nabla\right) \phi\right)+\frac{h^{e q}}{\rho R T}\left(c_{i}-u\right)\left(-\nabla \psi+\kappa \rho \nabla \nabla^{2} \rho\right) \\
& -\frac{h}{\rho}\left(c_{i}-u\right) \cdot \nabla \rho-h \nabla \cdot u
\end{aligned}
$$

The value of the phase field $(\phi)$ can be calculated by taking the zero moment from the distribution function $h_{\alpha}$.

$\phi=\Sigma h_{i}$

Now, after calculating $\phi$, we can calculate the value of $b$.

$b=\phi b_{\text {gas }}+(1-\phi) b_{\text {liq }}$

\subsection{Discretization}

In order to improve the stability and accuracy, the Eq. (1) is solved using the modified Lax-Wendroff method with the artificial viscosity [69]. The Lax-Wendroff scheme is very dispersive and shows strong oscillatory effects. Therefore, this method is not suitable for simulating the shock phenomenon. In order to eliminate the parasitic oscillations and increase the stability at high Mach numbers, the artificial viscosity term is added to the governing equations of the LBM [70]. Hence, the Eq. (1) can be written in the following form.

$\frac{\partial f_{k i}}{\partial t}+c_{k i} \cdot \frac{\partial f_{k i}}{\partial x}=-\frac{f_{k i}-f_{k i}^{e q}}{\lambda}+\frac{c_{k i}\left(1-\left(c_{k i} \frac{\Delta t}{\Delta x}\right)^{2}\right) \Delta x^{2}}{6} \frac{\partial^{3} f_{k i}}{\partial x^{3}}+$
$\left.\theta_{i}\left|u \frac{\Delta t}{\Delta x}\right|\left(1-\left|u \frac{\Delta t}{\Delta x}\right|\right) \frac{\Delta x^{2}}{2 \Delta t} \frac{\partial^{2} f_{k i}}{\partial x^{2}}+\frac{(\mathbf{c}-\mathbf{u}) \cdot(\mathbf{F}+\mathbf{G})}{\rho R T} f^{e q}\right]$

where

$\theta_{i}=\tau\left|\frac{P_{i+1}-2 P_{i}+P_{i-1}}{P_{i+1}+2 P_{i}+P_{i-1}}\right|$

In the above expression, $\theta_{i}$ is the switching function and $\tau$ represents the parameter controlling the artificial viscosity.

Assuming no gravitational force, the discretized equations for the distribution functions $f_{\alpha}$ and $h_{\alpha}$ could be acquired as shown below. It is worth mentioning that these equations are first order accurate in time and second order accurate in space.

$$
\begin{aligned}
f_{i}^{n+1} & =f_{i}^{n}-\frac{c_{i} \Delta \mathrm{t}}{2 \Delta \mathrm{x}}\left(f_{i+1}^{n}-f_{i-1}^{n}\right)-\frac{\Delta \mathrm{t}}{\lambda}\left(f_{i}^{n}-f_{i}^{e q}\right) \\
& +\frac{\left(\frac{c_{i} \Delta \mathrm{t}}{\Delta \mathrm{x}}\right)^{2}}{2}\left(f_{i+1}^{n}-2 f_{i}^{n}+f_{i-1}^{n}\right)+ \\
& \left(\frac{\left(\frac{c_{i} \Delta \mathrm{t}}{\Delta \mathrm{x}}\right)\left(1-\left(\frac{c_{i} \Delta \mathrm{t}}{\Delta \mathrm{x}}\right)^{2}\right)}{12}\right)\left(f_{i+1}^{n}-2 f_{i}^{n}+2 f_{i-1}^{n}-f_{i-2}^{n}\right) \\
& +\frac{\theta_{i}\left|u_{i} \frac{\Delta t}{\Delta x}\right|\left(1-\left|u_{i} \frac{\Delta t}{\Delta x}\right|\right)}{2}\left(f_{i+1}^{n}-2 f_{i}^{n}+f_{i-1}^{n}\right) \\
& +\frac{\Delta \mathrm{t}}{R} \frac{\Gamma_{i}}{T_{i}}\left(c_{i}-u_{i}\right) F_{i}
\end{aligned}
$$




$$
\begin{aligned}
h_{i}^{n+1} & =h_{i}^{n}-\frac{c_{i} \Delta \mathrm{t}}{2 \Delta \mathrm{x}}\left(h_{i+1}^{n}-h_{i-1}^{n}\right)+\frac{h_{i}}{\phi}\left(\phi_{i}^{n}-\phi_{i}^{n-1}\right) \\
& +\Delta \mathrm{t}\left(\frac{h_{i}}{\phi} \frac{\phi_{i+1}^{n}-\phi_{i-1}^{n}}{2 \Delta \mathrm{x}}-\frac{1}{\lambda}\left(h_{i}^{n}-h_{i}^{e q}\right)\right) \\
& +\Delta \mathrm{t}\left(\frac{1}{\rho} \frac{h_{i}^{e q}}{R T}\left(c_{i}-u\right) F_{i}-\frac{h_{i}}{\rho_{i}}\left(c_{i}-u\right) \frac{\rho_{i+1}^{n}-\rho_{i-1}^{n}}{2 \Delta \mathrm{x}}\right) \\
& +\Delta \mathrm{t}\left(h \frac{u_{i+1}^{n}-u_{i-1}^{n}}{2 \Delta \mathrm{x}}\right)
\end{aligned}
$$

\section{Solution and results}

Three major test cases are presented in this section to examine the accuracy of the proposed method.

\subsection{Sod shock tube problem}

The Sod shock tube problem is one of the most wellknown test cases to evaluate the accuracy of a compressible flow solver. This test is a one-dimensional Riemann problem. The fluid with high pressure and density exists in the left side and the fluid with low pressure and density is initialized at the right side. In the current problem, the left pressure and density is ten times higher than the original Sod problem [71] which leads to the pressure ratio of 100:1. The initial conditions are as follows $[3,72]$ :

$(\rho, \mathrm{u}, \mathrm{P}, \phi)= \begin{cases}(10,0,10,1) & x<0.5 \\ (0.125,0,0.1,0) & x>0.5\end{cases}$

Note that the initial temperature is calculated using the Van der Waals EOS (Eq. 4) and the initial values of pressure and density. The ratios of the specific heat capacity for the left and right sides are as $\gamma_{L}=1.4$ and $\gamma_{R}=1.6$, respectively.

Grid independence study has been performed and the second order accuracy has been achieved. Therefore, the problem is solved using $\Delta X=0.004$ and $\Delta t=0.000125$.

The results of the pressure, density, velocity and temperature at different times are shown in Figs. 2, 3, 4 and 5. Also for better comparison, the simulation results at $t=0.015$ hboxs are shown in Figs. 6, 7 and 8 and compared with the exact solutions obtained from Ahmed et al. [3]. As can be seen, the simulation results agree well with the exact solutions.

\subsection{Two fluids combination problem}

In this test case, the initial conditions are as follows [3]:

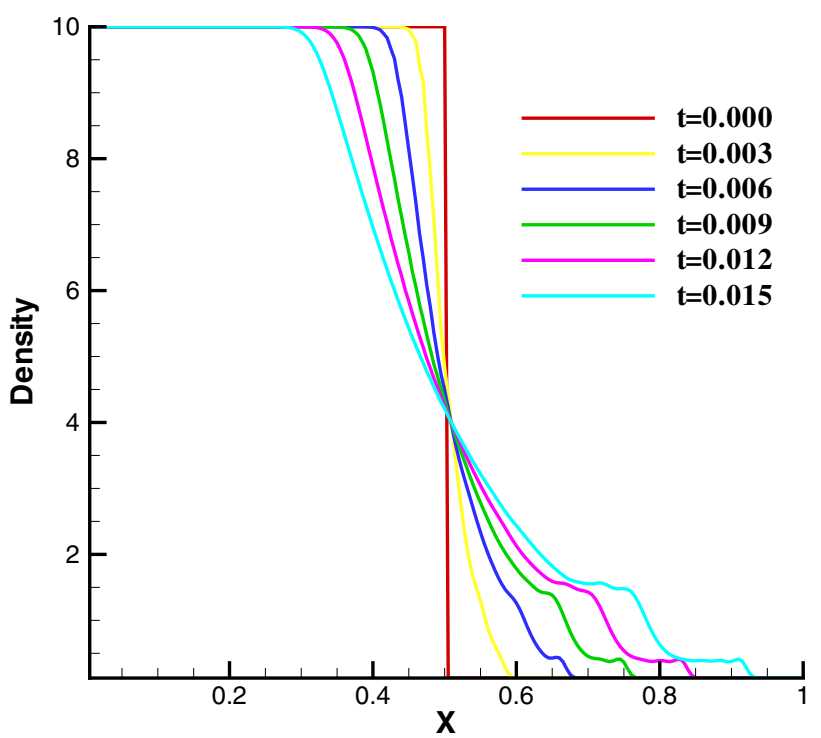

Fig. 2 Density changes at different times

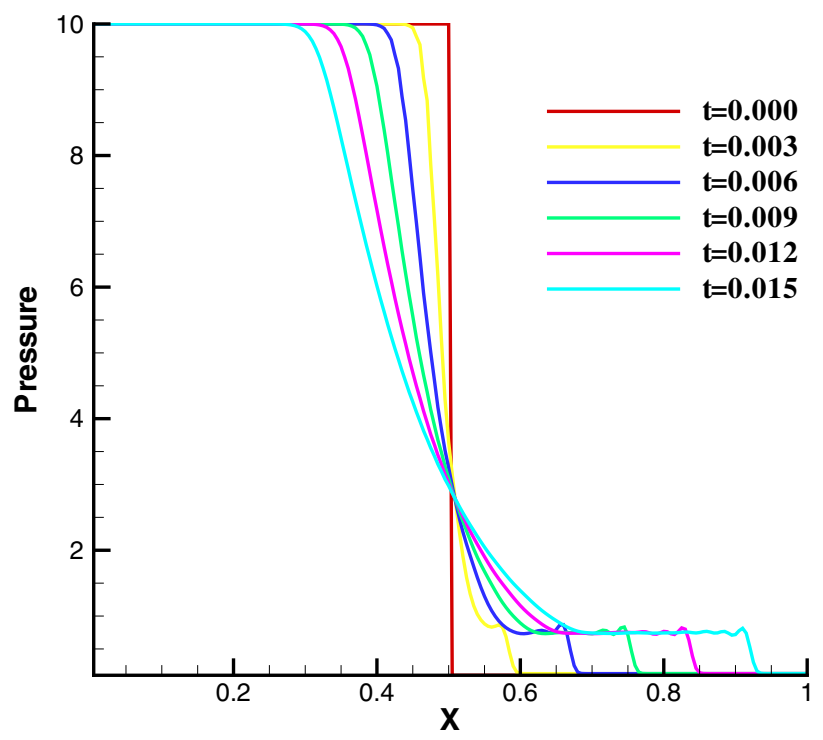

Fig. 3 Pressure changes at different times

$(\rho, \mathrm{u}, \mathrm{P}, \phi)= \begin{cases}(2.0,0,1000,1) & x<0.5 \\ (1.0,0,0.01,0) & x>0.5\end{cases}$

Note that the ratios of the specific heat capacity for the left and right sides are as $\gamma_{L}=1.4$ and $\gamma_{R}=1.2$, respectively. The simulation results at $t=0.015 \mathrm{~s}$ are given in Figs. 9,10 and 11 and compared with the exact solutions obtained from Ahmed et al. [3]. Similar to the previous test case, the agreement between the simulation results and exact solutions are satisfactory. 


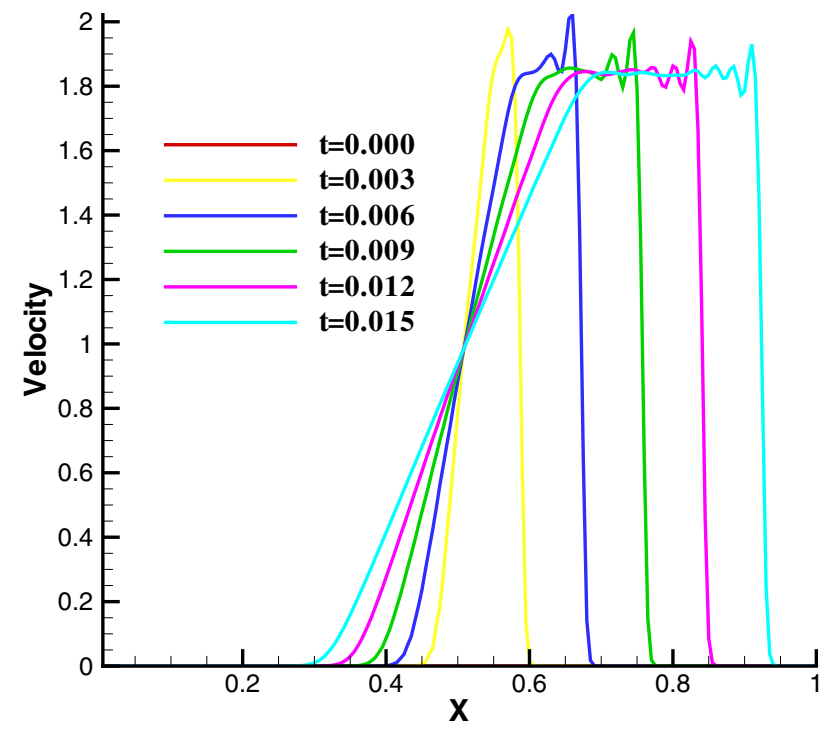

Fig. 4 Velocity changes at different times

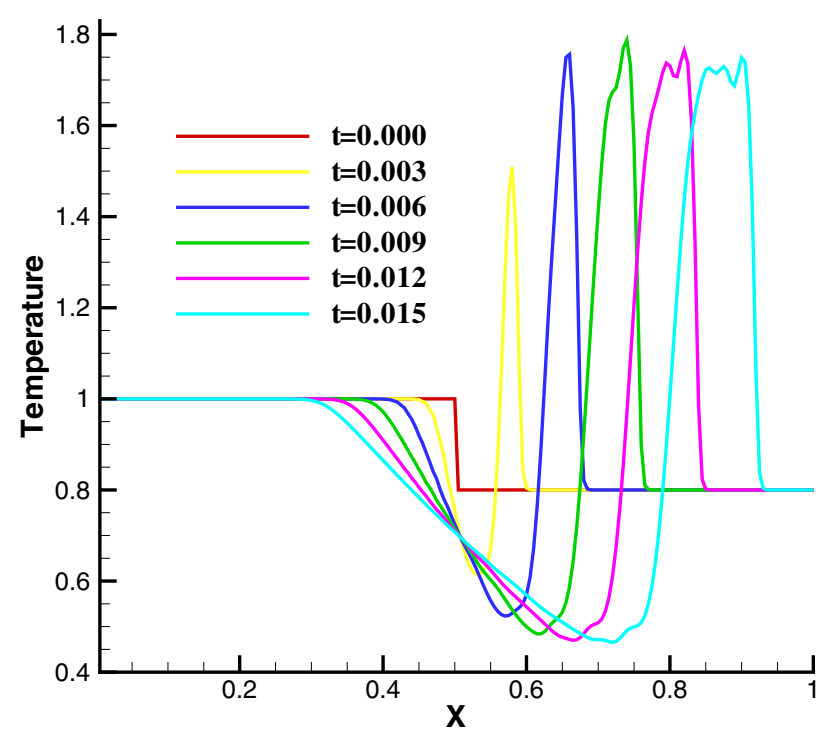

Fig. 5 Temperature changes at different times

\subsection{Water-air mixture problem}

For the last test case, a 1-m long shock tube containing two chambers separated by an interface at the location $x=0.8 \mathrm{~m}$ is considered. Each chamber contains a mixture of water and air. The initial density of the water is set to $\rho_{\text {water }}=1000 \mathrm{~kg} \mathrm{~m}^{-3}$ and the Stiffened equation of state (EOS) with the parameter $\gamma_{\text {water }}=4.4$ is employed. The initial density of air is considered to be $\rho_{\text {air }}=10 \mathrm{~kg} \mathrm{~m}^{-3}$ and EOS parameter is assumed to be $\gamma_{\text {air }}=1.4$. The left chamber contains a very small volume fraction of air $\phi_{\text {air }}=10^{-6}$ with the pressure $10^{9} \mathrm{~Pa}$. The right chamber contains the

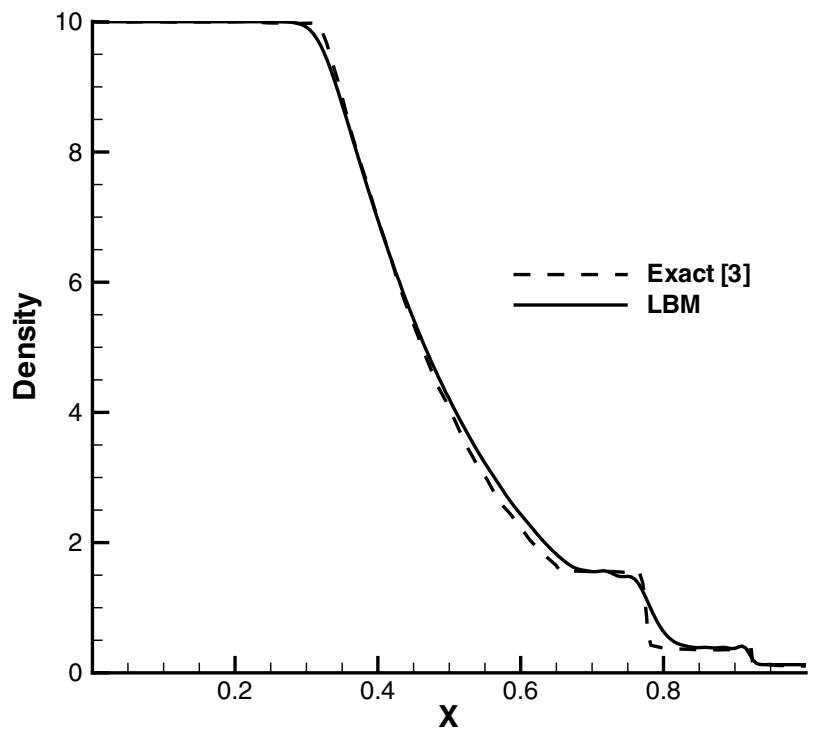

Fig. 6 Density changes and comparison with the exact solution (from Ahmed et al. [3])

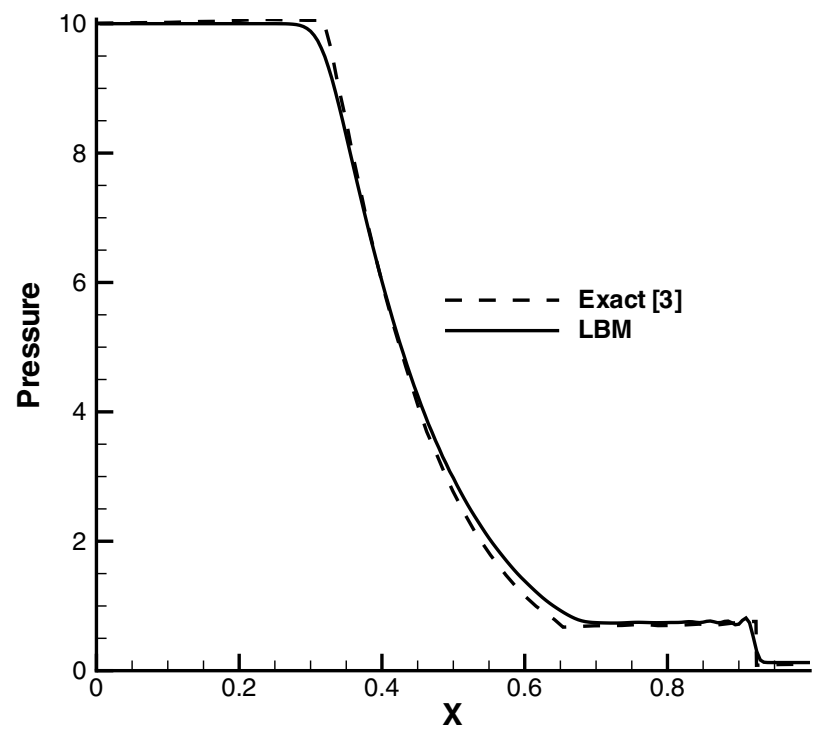

Fig. 7 Pressure changes and comparison with the exact solution (from Ahmed et al. [3])

same fluids but the volume fractions are reversed with the pressure $10^{5} \mathrm{~Pa}$. The fluids are initially at rest in both chambers. [4].

In summary, the initial conditions are as follows:

$(\rho, \mathrm{U}, \mathrm{P}, \phi)= \begin{cases}(1000,0,1000,1) & x<0.8 \\ (10,0,0.01,0) & x>0.8\end{cases}$

The simulation results at $t=0.220 \mathrm{~s}$ are shown in Figs. 12, 13 and 14 and compared with the exact solutions obtained 


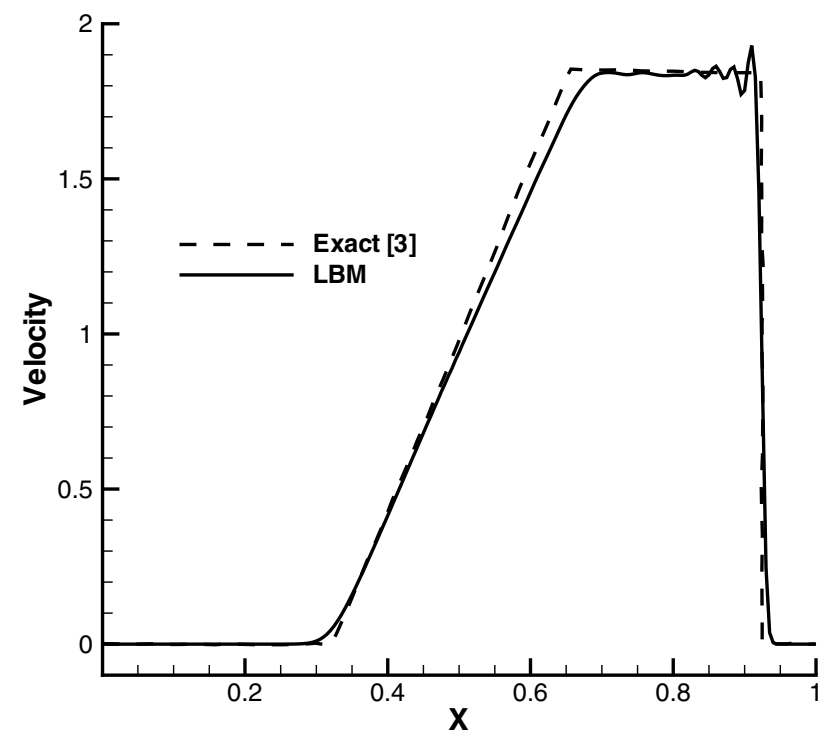

Fig. 8 Velocity changes and comparison with the exact solution (from Ahmed et al. [3])

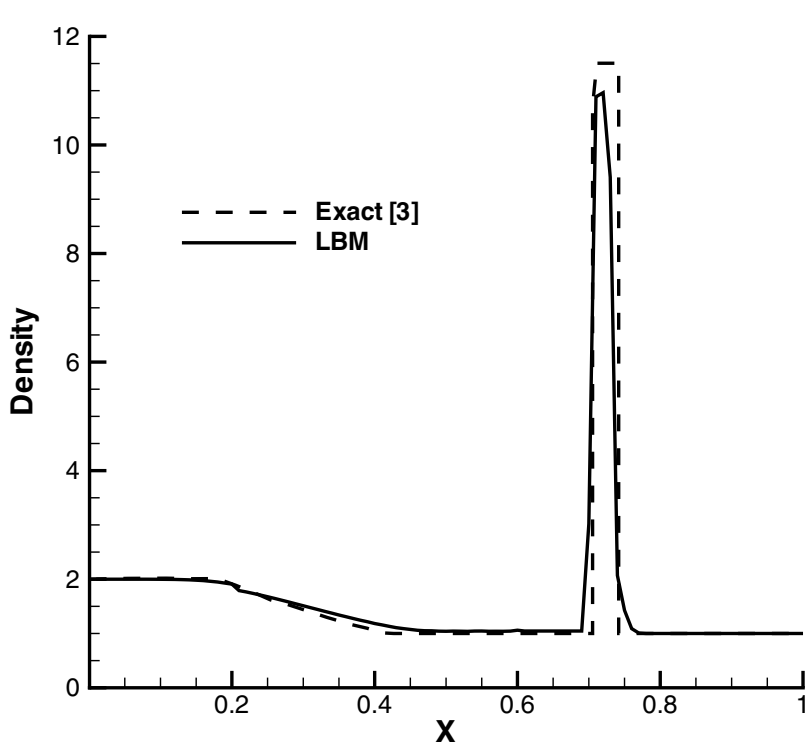

Fig. 9 Density changes and comparison with the exact solution (from Ahmed et al. [3])

from Saurel et al. [4]. As observed, the simulation results correspond well with the exact solutions which confirms the accuracy and consistency of the proposed method. Figure 15 shows the liquid volume fraction. As illustrated, the phase interface between the water and the air moves a little bit more to the left, but still corresponds well with the exact solution.

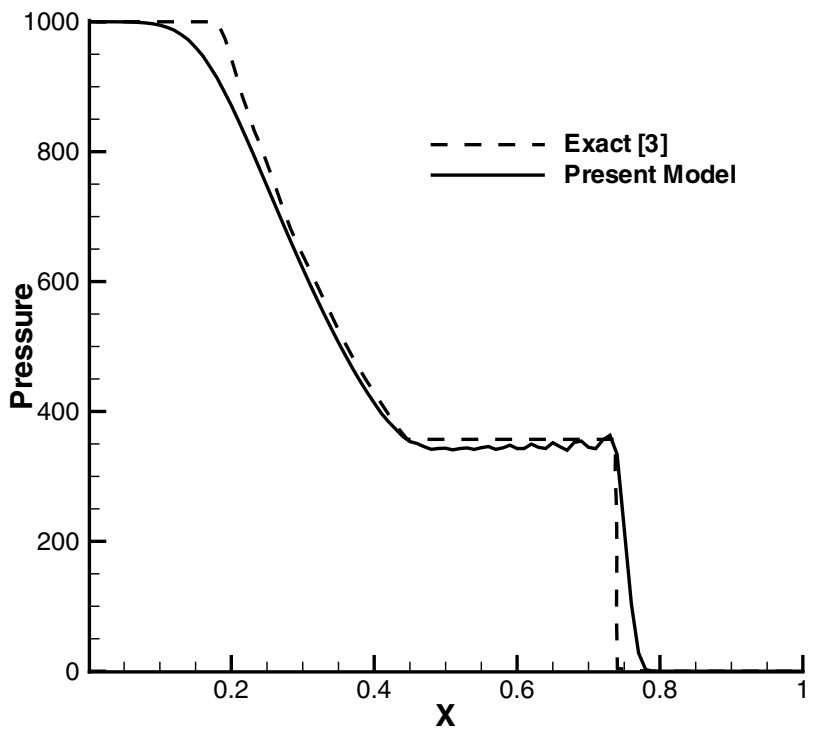

Fig. 10 Pressure changes and comparison with the exact solution (from Ahmed et al. [3])

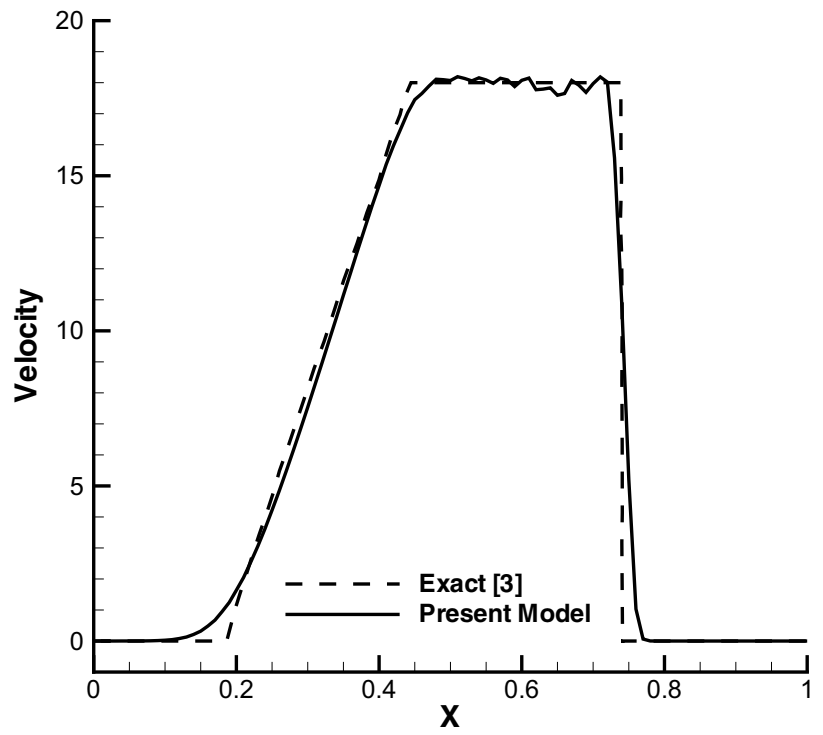

Fig. 11 Velocity changes and comparison with the exact solution (from Ahmed et al. [3])

\subsection{Water-air mixture problem with high density ratio}

One of the remarkable features of the proposed method is the ability to model a two-phase compression problem with a high density ratio. In order to demonstrate this, the problem of the previous section is solved with a high density ratio of 660 . Therefore, the initial conditions are considered as: 


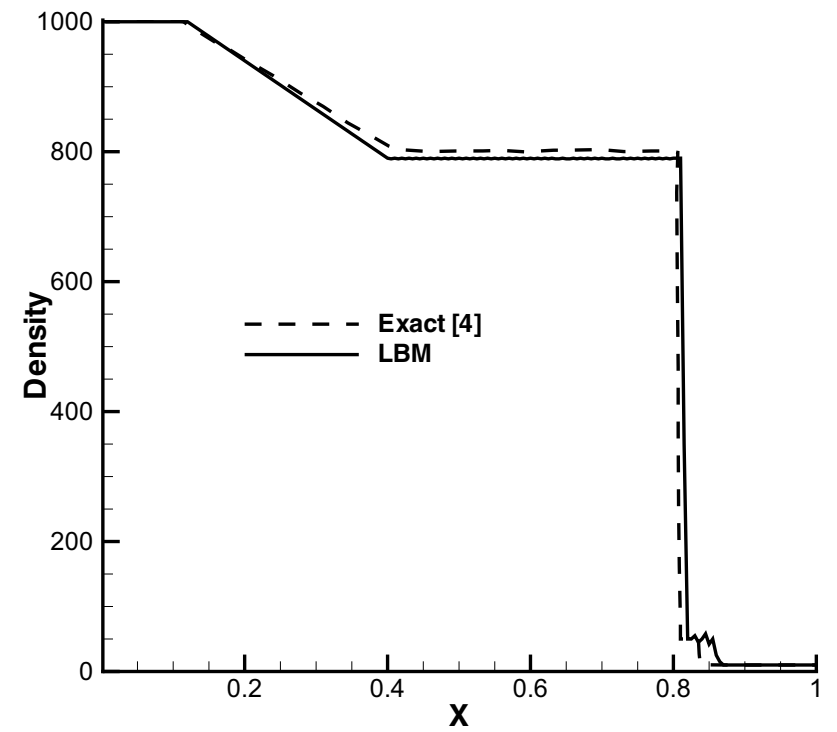

Fig. 12 Density changes and comparison with the exact solution (from Saurel et al. [4])

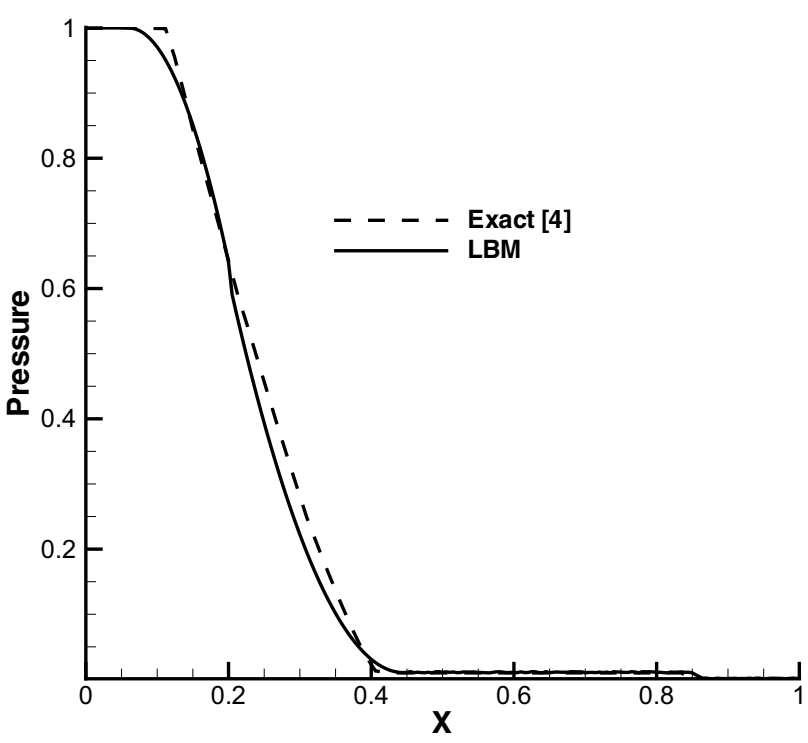

Fig. 13 Pressure changes and comparison with the exact solution (from Saurel et al. [4])

$(\rho, \mathrm{u}, \mathrm{P}, \phi)= \begin{cases}(1000,0,1000,1) & x<0.8 \\ (1.5,0,0.01,0) & x>0.8\end{cases}$

The diagrams of the variations in density, pressure, velocity and the composition along the shock tube at $t=0.220 \mathrm{~s}$ are shown in Figs. 16, 17, 18 and 19. As observed, the stable solution can be acquired at high density ratios. Similar to the previous test case, the interface location is almost unchanged relative to the initial condition.

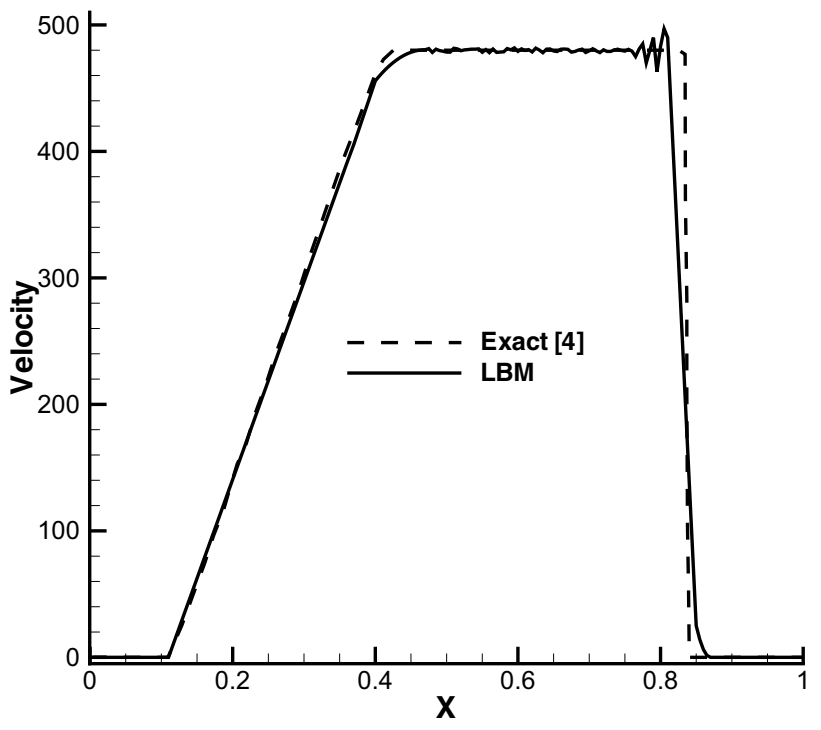

Fig. 14 Velocity changes and comparison with the exact solution (from Saurel et al. [4])

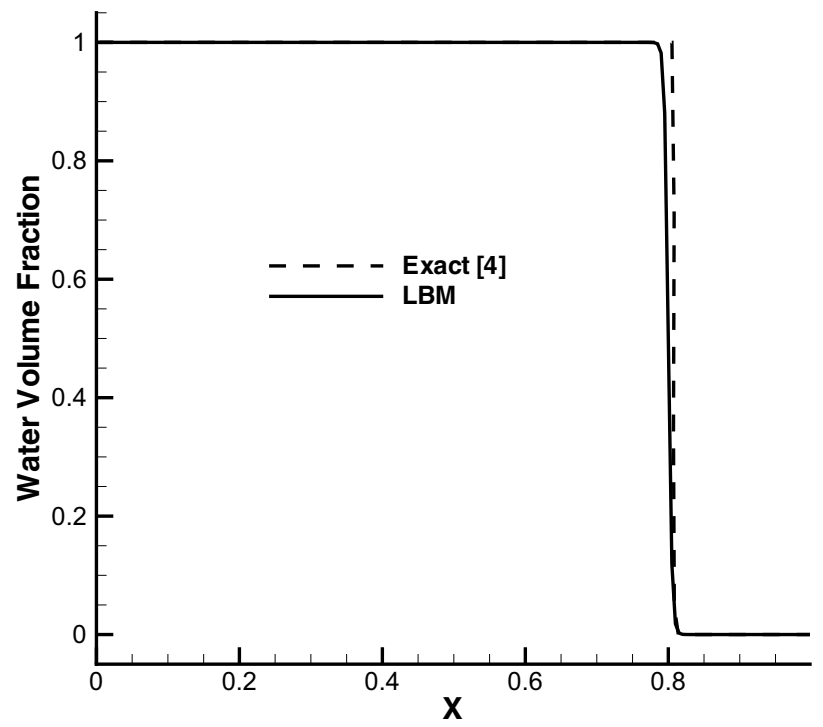

Fig. 15 Liquid volume fraction and comparison with the exact solution (from Saurel et al. [4])

\section{Discussion}

To the best of our knowledge, the LB simulation of two-phase compressible flows using the phase-field approach has not been addressed in the literature before. As mentioned in the introduction, almost all of the literature available on the cavitation and compressible flow calculations within two-phase fluid flows using LBM are devoted to the pseudopotential models $[56,57]$. In this work, another approach is proposed to study the 


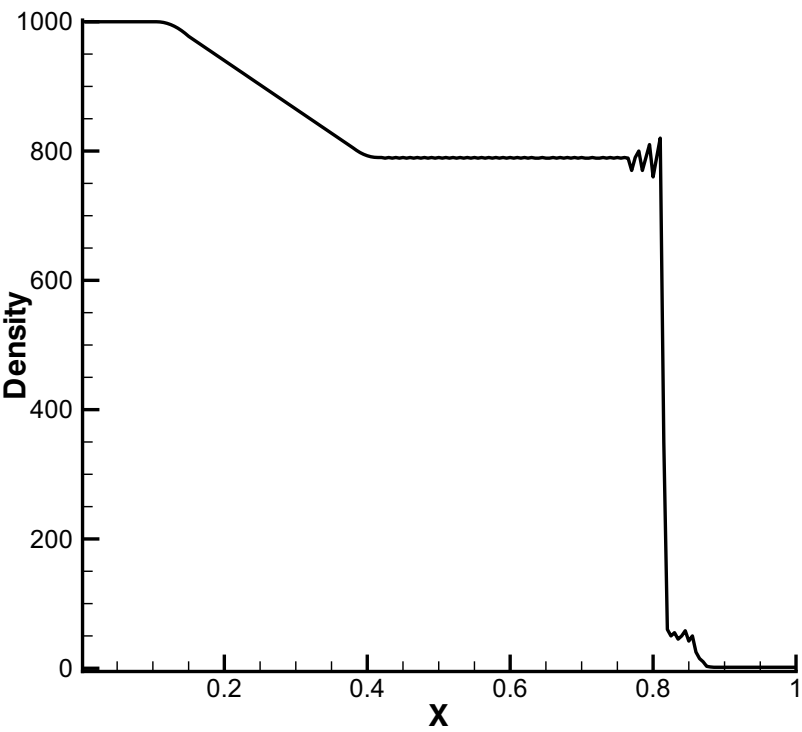

Fig. 16 Density variations along the tube

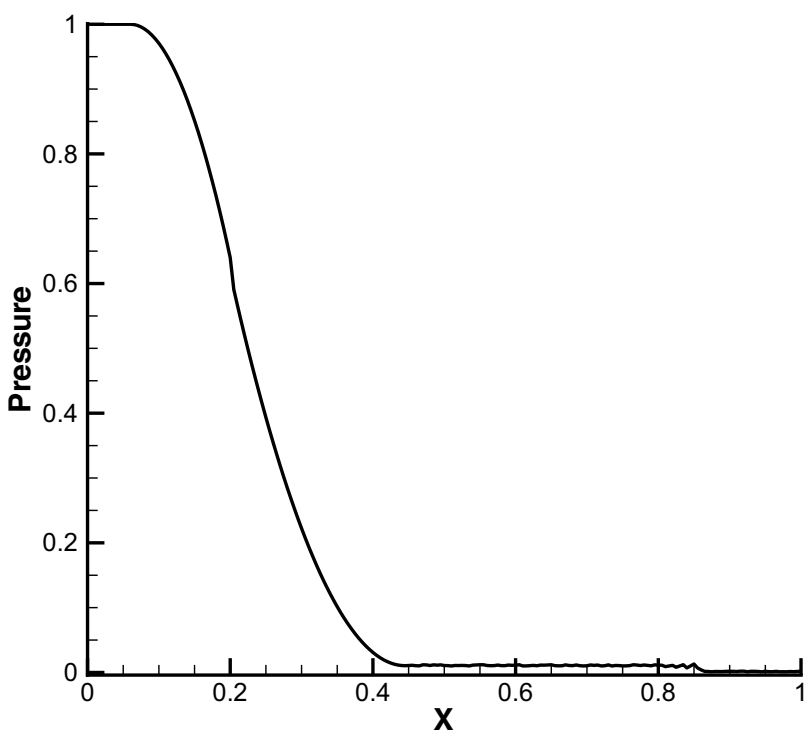

Fig. 17 Pressure variations along the tube

compressible two-phase flow in general. Our motivation was to overcome the deficiencies of the pseudopotential models in which the external and inter-molecular forces are implemented using potential functions. Hence, the fluid properties (including density ratio and surface tension) are indirectly set through preliminary calculations using the developed code. In our model, the forces are introduced directly into the discrete Boltzmann equation (DBE), so that the fluid properties such as density ratio and surface tension can be directly and freely adjusted. The DBE is later discretized and solved using a finite

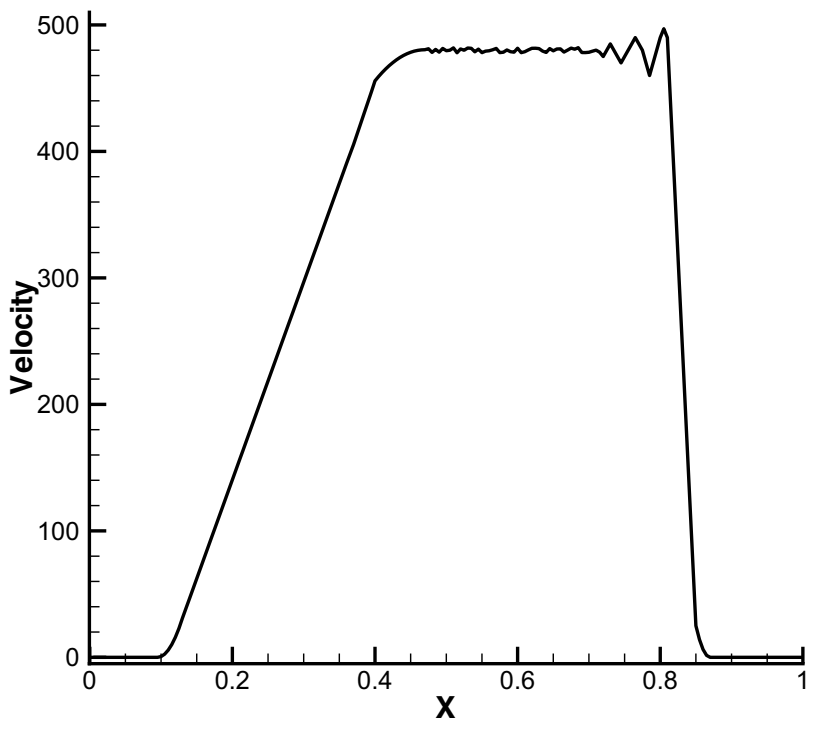

Fig. 18 Velocity variations along the tube

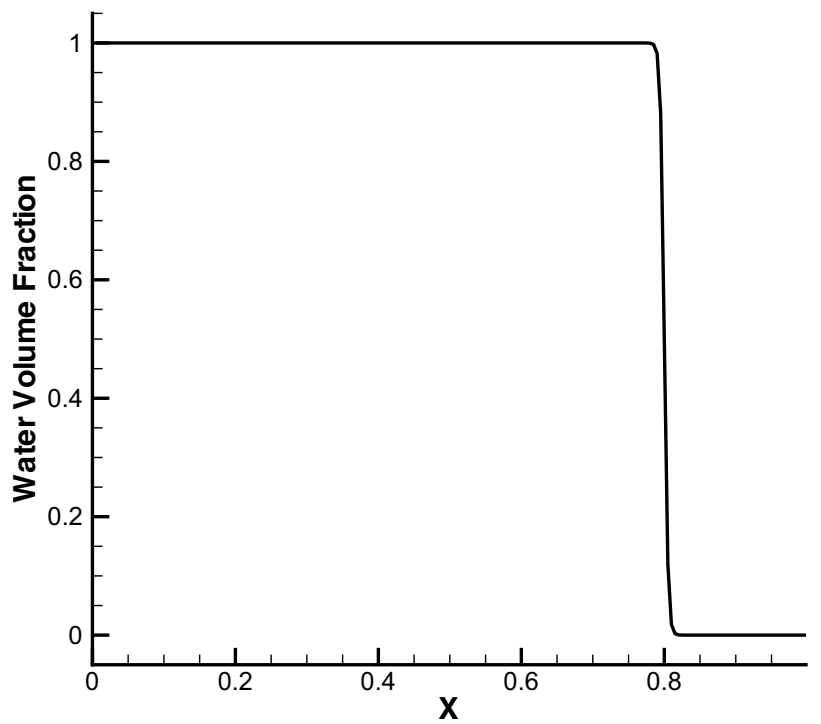

Fig. 19 Liquid volume fraction along the tube

difference scheme. Moreover, a modified equilibrium distribution function is incorporated to consistently recover the fully compressible Navier-Stokes equations.

The presented model is validated through various onedimensional test cases. First, the one-dimensional Sod shock tube problem was simulated in order to validate our multiphase compressible solver. Subsequently, the accuracy of the model against two-phase compressible flows was examined. Employing the developed framework, we managed to consistently simulate the one-dimensional two-phase shock tube problem using LBM for the first time. Furthermore, we have demonstrated that the developed model remains stable at high density ratios up to 
1:1000. The benchmark simulations have been successfully validated against the reference solutions given by Ahmed [3] and Saurel [4].

\section{Conclusion}

One-dimensional D1Q5 lattice Boltzmann method was developed for the numerical simulation of compressible two-phase flows. The compressible single-phase LB solver proposed by Kataoka [42] was extended to take into account the phase separation processes, so that the computation of a two-phase compressible flow is allowed. The phase separation was achieved by considering the intermolecular forcing term computed by the method of He et al. [58]. In order to capture the phase interface, the Cahn-Hilliard phase-field equation was solved throughout the computational domain. A separate distribution function was introduced in order to solve the Cahn-Hilliard within the lattice Boltzmann framework. In order to confirm the accuracy and consistency of the proposed method, several one-dimensional problems were simulated and compared with the exact solutions. A test case with high density ratio was also presented. As shown, the initial results presented in this article were promising and demonstrated the capability of the proposed model to consistently simulate the compressible two-phase fluid flows with a straightforward routine. The future works will focus on the application of this method in the modeling of cavitation phenomena in two and three dimensions.

\section{Compliance with ethical standards}

Conflict of interest The authors declare that they have no conflict of interest.

\section{References}

1. Zhang L-X, Zhang N, Peng X-X, Wang B-L, Shao X-M (2015) A review of studies of mechanism and prediction of tip vortex cavitation inception. J Hydrodyn Ser B 27(4):488-495

2. Yazdi H, Rahimiani MH, Safari $H$ (2018) Numerical simulation of pressure-driven phase-change in two-phase fluid flows using the lattice Boltzmann method. Comput Fluids 172:8-18

3. Ahmed M, Saleem MR, Zia S, Qamar S (2015) Central upwind scheme for a compressible two-phase flow model. PLoS ONE 10(6):e0126273

4. Saurel R, Petitpas F, Berry RA (2009) Simple and efficient relaxation methods for interfaces separating compressible fluids, cavitating flows and shocks in multiphase mixtures. J Comput Phys 228(5):1678-1712
5. Akhtar MW, Kleis SJ (2013) Boiling flow simulations on adaptive octree grids. Int J Multiph Flow 53:88-99

6. Sato Y, Ničeno B (2013) A sharp-interface phase change model for a mass-conservative interface tracking method. J Comput Phys 249:127-161

7. Shu B, Dammel F, Stephan P (2008) Phase change model for two-phase fluid flow based on the volume of fluid method. In: ICHMT Digital Library Online, Begel House Inc.

8. Gibou F, Chen L, Nguyen D, Banerjee S (2007) A level set based sharp interface method for the multiphase incompressible Navier-Stokes equations with phase change. J Comput Phys 222(2):536-555

9. Luo X-Y, Ni M-J, Ying A, Abdou M (2005) Numerical modeling for multiphase incompressible flow with phase change. Numer Heat Transf Part B Fundam 48(5):425-444

10. Son G, Dhir VK (1998) Numerical simulation of film boiling near critical pressures with a level set method. J Heat Transf 120(1):183-192

11. Tryggvason G, Bunner B, Esmaeeli A, Juric D, Al-Rawahi N, Tauber W, Han J, Nas S, Jan Y-J (2001) A front-tracking method for the computations of multiphase flow. J Comput Phys 169(2):708-759

12. Saurel R, Lemetayer O (2001) A multiphase model for compressible flows with interfaces, shocks, detonation waves and cavitation. J Fluid Mech 431:239-271

13. Chang $\mathrm{C}-\mathrm{H}$, Liou M-S (2007) A robust and accurate approach to computing compressible multiphase flow: stratified flow model and AUSM+-up scheme. J Comput Phys 225(1):840-873

14. Johnsen E, Colonius $T$ (2006) Implementation of weno schemes in compressible multicomponent flow problems. J Comput Phys 219(2):715-732

15. Johnsen $E$ (2008) Spurious oscillations and conservation errors in interface-capturing schemes. Annu Res Briefs 2008:115-126

16. Kawai S, Terashima H (2011) A high-resolution scheme for compressible multicomponent flows with shock waves. Int J Numer Methods Fluids 66(10):1207-1225

17. Shyue K-M (1998) An efficient shock-capturing algorithm for compressible multicomponent problems. J Comput Phys 142(1):208-242

18. Shyue K-M (2010) A high-resolution mapped grid algorithm for compressible multiphase flow problems. J Comput Phys 229(23):8780-8801

19. Abgrall $R$ (1996) How to prevent pressure oscillations in multicomponent flow calculations: a quasi conservative approach. J Comput Phys 125(1):150-160

20. Saurel R, Abgrall R (1999) A multiphase Godunov method for compressible multifluid and multiphase flows. J Comput Phys 150(2):425-467

21. Castro C, Toro E (2006) A riemann solver and upwind methods for a two-phase flow model in non-conservative form. Int $J$ Numer Methods Fluids 50(3):275-307

22. Dumbser M, Toro EF (2011) A simple extension of the osher riemann solver to non-conservative hyperbolic systems. J Sci Comput 48(1-3):70-88

23. Munkejord ST (2010) A numerical study of two-fluid models with pressure and velocity relaxation. Adv Appl Math Mech 2:131-159

24. Munkejord ST (2007) Comparison of Roe-type methods for solving the two-fluid model with and without pressure relaxation. Comput Fluids 36(6):1061-1080

25. Tokareva S, Toro EF (2010) HLLC-type Riemann solver for the Baer-Nunziato equations of compressible two-phase flow. $J$ Comput Phys 229(10):3573-3604

26. Kapila A, Menikoff R, Bdzil J, Son S, Stewart DS (2001) Two-phase modeling of deflagration-to-detonation 
transition in granular materials: reduced equations. Phys Fluids 13(10):3002-3024

27. Murrone A, Guillard H (2005) A five equation reduced model for compressible two phase flow problems. J Comput Phys 202(2):664-698

28. Daude F, Galon P, Gao Z, Blaud E (2014) Numerical experiments using a HLLC-type scheme with ale formulation for compressible two-phase flows five-equation models with phase transition. Comput Fluids 94:112-138

29. Kokh S, Lagoutiere F (2010) An anti-diffusive numerical scheme for the simulation of interfaces between compressible fluids by means of a five-equation model. J Comput Phys 229(8):2773-2809

30. Kreeft JJ, Koren B (2010) A new formulation of Kapilas fiveequation model for compressible two-fluid flow, and its numerical treatment. J Comput Phys 229(18):6220-6242

31. Qamar S, Ahmed M (2009) A high order kinetic flux-vector splitting method for the reduced five-equation model of compressible two-fluid flows. J Comput Phys 228(24):9059-9078

32. Ansari M, Daramizadeh A (2013) Numerical simulation of compressible two-phase flow using a diffuse interface method. Int J Heat Fluid Flow 42:209-223

33. Farokhirad S, Lee T, Morris JF (2013) Effects of inertia and viscosity on single droplet deformation in confined shear flow. Commun Comput Phys 13(3):706-724

34. Farokhirad S, Morris JF, Lee T (2015) Coalescence-induced jumping of droplet: inertia and viscosity effects. Phys Fluids 27(10):102102

35. He X, Shan X, Doolen GD (1998) Discrete Boltzmann equation model for nonideal gases. Phys Rev E 57(1):R13

36. Zhang R, Farokhirad S, Lee T, Koplik J (2014) Multiscale liquid drop impact on wettable and textured surfaces. Phys Fluids 26(8):082003

37. Amirshaghaghi $H$, Rahimian $M$, Safari $H$ (2016) Application of a two phase lattice Boltzmann model in simulation of free surface jet impingement heat transfer. Int Commun Heat Mass Transf 75:282-294

38. Amirshaghaghi $\mathrm{H}$, Rahimian $\mathrm{MH}$, Safari $\mathrm{H}$, Krafczyk M (2018) Large Eddy simulation of liquid sheet breakup using a twophase lattice Boltzmann method. Comput Fluids 160:93-107

39. Guangwu Y, Yaosong C, Shouxin H (1999) Simple lattice Boltzmann model for simulating flows with shock wave. Phys Rev E 59(1):454

40. Shi W, Shyy W, Mei R (2001) Finite-difference-based lattice Boltzmann method for inviscid compressible flows. Numer Heat Transf Part B Fundam 40(1):1-21

41. Kataoka T, Tsutahara M (2004) Lattice Boltzmann method for the compressible Euler equations. Phys Rev E 69(5):056702

42. Kataoka T, Tsutahara M (2004) Lattice Boltzmann model for the compressible Navier-Stokes equations with flexible specificheat ratio. Phys Rev E 69(3):035701

43. Sun C, Hsu A (2004) Multi-level lattice Boltzmann model on square lattice for compressible flows. Comput Fluids 33(10):1363-1385

44. Sun C, Hsu AT (2003) Three-dimensional lattice Boltzmann model for compressible flows. Phys Rev E 68(1):016303

45. Sun C (2000) Simulations of compressible flows with strong shocks by an adaptive lattice Boltzmann model. J Comput Phys 161(1):70-84

46. Sun C (1998) Lattice-Boltzmann models for high speed flows. Phys Rev E 58(6):7283

47. Sun C (2000) Adaptive lattice Boltzmann model for compressible flows: viscous and conductive properties. Phys Rev E $61(3): 2645$
48. Watari M, Tsutahara M (2006) Supersonic flow simulations by a three-dimensional multispeed thermal model of the finite difference lattice Boltzmann method. Physica A 364:129-144

49. Watari M, Tsutahara M (2003) Two-dimensional thermal model of the finite-difference lattice Boltzmann method with high spatial isotropy. Phys Rev E 67(3):036306

50. Watari M, Tsutahara M (2004) Possibility of constructing a multispeed Bhatnagar-Gross-Krook thermal model of the lattice Boltzmann method. Phys Rev E 70(1):016703

51. Watari M (2007) Finite difference lattice Boltzmann method with arbitrary specific heat ratio applicable to supersonic flow simulations. Physica A 382(2):502-522

52. Qu K, Shu C, Chew Y (2007) Alternative method to construct equilibrium distribution functions in lattice-Boltzmann method simulation of inviscid compressible flows at high Mach number. Phys Rev E 75(3):036706

53. Qu K, Shu C, Chew YT (2007) Simulation of shock-wave propagation with finite volume lattice Boltzmann method. Int J Mod Phys C 18(04):447-454

54. He Y-L, Liu Q, Li Q (2013) Three-dimensional finite-difference lattice Boltzmann model and its application to inviscid compressible flows with shock waves. Physica A 392(20):4884-4896

55. Gunstensen AK, Rothman DH, Zaleski S, Zanetti G (1991) Lattice Boltzmann model of immiscible fluids. Phys Rev A 43(8):4320

56. Shan X, Chen H (1993) Lattice Boltzmann model for simulating flows with multiple phases and components. Phys Rev $E$ 47(3): 1815

57. Shan X, Chen H (1994) Simulation of nonideal gases and liquidgas phase transitions by the lattice Boltzmann equation. Phys Rev E 49(4):2941

58. He X, Chen S, Zhang R (1999) A lattice Boltzmann scheme for incompressible multiphase flow and its application in simulation of Rayleigh-Taylor instability. J Comput Phys 152(2):642-663

59. Lee T, Lin C-L (2003) Pressure evolution lattice-Boltzmann-equation method for two-phase flow with phase change. Phys Rev $E$ 67(5):056703

60. Zhang R, Chen H (2003) Lattice Boltzmann method for simulations of liquid-vapor thermal flows. Phys Rev E 67(6):066711

61. Lee $T$, Liu L (2010) Lattice Boltzmann simulations of micron-scale drop impact on dry surfaces. J Comput Phys 229(20):8045-8063

62. Lee T (2009) Effects of incompressibility on the elimination of parasitic currents in the lattice Boltzmann equation method for binary fluids. Comput Math Appl 58(5):987-994

63. Safari H, Rahimian MH, Krafczyk M (2013) Extended lattice Boltzmann method for numerical simulation of thermal phase change in two-phase fluid flow. Phys Rev E 88(1):013304

64. Safari H, Rahimian MH, Krafczyk M (2014) Consistent simulation of droplet evaporation based on the phase-field multiphase lattice Boltzmann method. Phys Rev E 90(3):033305

65. Mohammadi-Shad M, Lee T (2017) Phase-field lattice Boltzmann modeling of boiling using a sharp-interface energy solver. Phys Rev E 96(1):013306

66. Begmohammadi A, Farhadzadeh M, Rahimian MH (2015) Simulation of pool boiling and periodic bubble release at high density ratio using lattice Boltzmann method. Int Commun Heat Mass Transf 61:78-87

67. Sadeghi R, Shadloo MS, Jamalabadi MYA, Karimipour A (2016) A three-dimensional lattice Boltzmann model for numerical investigation of bubble growth in pool boiling. Int Commun Heat Mass Transf 79:58-66

68. Rowlinson JS, Widom B (2013) Molecular theory of capillarity. Courier Corporation, Chelmsford

69. Gan Y, Xu A, Zhang G, Yu X, Li Y (2008) Two-dimensional lattice Boltzmann model for compressible flows with high Mach number. Physica A 387(8-9):1721-1732 
70. Hirsh C (1990) Numerical computation of internal and external flows. Computational methods for inviscid and viscous flows. Wiley, Chichester

71. Sod GA (1978) A survey of several finite difference methods for systems of nonlinear hyperbolic conservation laws. J Comput Phys 27(1):1-31
72. Wackers J, Koren B (2004) Five-equation model for compressible two-fluid flow. Modelling, Analysis and Simulation [MAS] (E 0414)

Publisher's Note Springer Nature remains neutral with regard to jurisdictional claims in published maps and institutional affiliations. 